\title{
Mesoporous Silica Nanoparticles (MSN): a nanonetwork and hierarchical structure in drug delivery
}

\begin{abstract}
Nanotechnology is a fast-growing area, involving the fabrication and use of nano-sized materials and devices. Nanocomposite materials play a number of important roles in modern science and technology, including pharmaceutical science. Mesoporous materials in particular have a large number of applications. In the past decade, mesoporous silica nanoparticles (MSNs) attracted attention increasingly for their potential biomedical applications. With their tailored mesoporous structure and high surface area, MSNs have significant advantages as drug delivery systems (DDSs) compared with traditional drug nanocarriers. Inorganic mesoporous materials are being used increasingly in pharmaceutical materials research to enhance the dissolution and permeation behavior of drugs that are poorly soluble in water. The benefits of using mesoporous materials in drug delivery applications stem from their large surface area and pore volume. These properties enable the materials to accommodate large amounts of payload molecules, protect them from premature degradation and promote controlled and fast release. As carriers with various morphologies and chemical surface properties can be produced, these materials may even promote adsorption from the gastrointestinal tract to the systemic circulation. In this work, we review recent progress in the synthesis and surface functionalization of MSNs for drug delivery applications.
\end{abstract}

Keywords: Mesoporous silica, SBA-15, Liquid crystal mechanism
Volume 2 Issue 5 - 2015

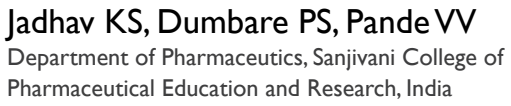

Pharmaceutical Education and Research, India

\begin{abstract}
Correspondence: Pande VV, Associate Professor and Head, Department of Pharmaceutics (PG), SanjivaniCollege of Pharmaceutical Education and Research, Kopargaon, Maharashtra, India, Tel 02423-285626, Email drvishalpande@gmail.com
\end{abstract}

Received: November 30, 2015 | Published: December 29, 2015
Abbreviations: MSNs, Mesoporous Silica Nanoparticles; DDSs, Drug Delivery Systems; SBA-15, Santa Barbara Amorphous-15; MCM-48, Mobile Crystalline Matter-48; CMC, Critical Micellar Concentration; TEOS, Tetraethoxysilane; CDA, Cytidine Deaminase

\section{Introduction}

According to IUPAC nomenclature, mesoporous materials are those materials having a pore size ranging from 2 to $50 \mathrm{~nm}$ in diameter. ${ }^{1-6}$ Mesoporous silica nanoparticles (MSNs) are promising and novel drug vehicles due to their unique mesoporous structure, which preserve a level of chemical stability, surface functionality and biocompatibility and ensure controlled release and targeted delivery of a variety of drug molecules. MSNs were discovered in 1992 by the Mobile Oil Corporation. They have received considerable attention due to their outstanding features, such as a high surface area, large pore volume, tunable pore diameter, narrow pore size distribution, ${ }^{7-15}$ easily modified surface, ${ }^{16}$ good biocompatibility and thermal stability. ${ }^{17}$

The word pore comes from the Greek word $\pi$ opo $\sigma$, which means 'passage'. This refers to pores acting as passages between the external and internal surfaces of a solid, allowing material to pass into, through, or out of the solid. ${ }^{16}$ The possibility of using mesoporous materials such as Santa Barbara Amorphous-15 (SBA-15) or Mobile Crystalline Matter-48 (MCM-48) and some metal organic frameworks as drug carriers and controlled release systems has been discussed. ${ }^{18,19}$ Mesoporous materials are essentially prepared through the formation of silica around template micelle assemblies followed by removal of the template using appropriate methods such as calcination and solvent evaporation. The most widely used mesoporous material is Santa Barbara Amorphous-15 (SBA-15), which has highly ordered pores with thick walls and a two-dimensional hexagonal structure. SBA-15 is produced using an amphiphilic triblock-copolymer of poly(ethylene oxide) and poly(propylene oxide) (PluronicP123) as the structure-directing reagent in highly acidic media. ${ }^{20} \mathrm{MSNs}$ are promising platforms for a diverse range of biomedical applications including bioimaging for diagnostics ${ }^{21}$ bone repair and scaffold engineering, drug delivery, ${ }^{22,23}$ biosensing $^{24}$ and biocatalysis or catalysis. ${ }^{25}$ Echocardiography can be used to avoid implantation into fibrotic tissue, but cellular imaging for surgical guidance is limited by the lack of available contrast agents. Paul J Kempen et al. ${ }^{26}$ proposed an MSN that solves both these major limitations through a combined therapeutic and diagnostic - "theranostic"-approach. Silica-based nanoparticles offer contrast for ultrasound imaging. ${ }^{26}$ MSNs are biocompatible, preferentially accumulate in tumors and effectively deliver drugs to tumors and suppress tumor growth. MSNs could be used as drug delivery vehicles, gene-transfection reagents, cell markers and carriers of molecules. ${ }^{27}$

\section{Chemistry, synthesis and functionalization of MSNs}

With an abundant availability of various types of surfactants and the development of a deep understanding of sol-gel chemistry, MSNs with different structures have been developed. The size, morphology, pore size and pore structure of MSNs can be rationally designed and the synthesis process can be freely controlled. ${ }^{28}$ As a consequence of the templating method employed for synthesis, the porosity properties of ordered mesoporous materials depend on the type of surfactant employed during the liquid crystal templating mechanism. ${ }^{29-31}$ More specifically, the synthetic process is based on the dissolution of surfactant molecules into polar solvents to yield so called "liquid crystals". The amount of surfactant dissolved in the solution plays a very important role during the synthetic process and when the concentration is above the critical micellar concentration $(\mathrm{cmc})$, the surfactant molecules aggregate to form micelles. Obviously, the characteristics of these micelles depend on the nature of the surfactant used and/or the experimental conditions, for example the 
temperature, $\mathrm{pH}$ and concentration. The micelles aggregate to yield supramicellar structures with determined geometries, which again depend on the chemical nature of the surfactant and the processing conditions. The resulting mesoporous framework depends on these supramicellar geometries, which may be hexagonal, cubic, or laminar. Once the supramicellar aggregates are formed, the silica precursors, normally alkoxysilanes are added to the liquid crystals. As a result of the sol-gel chemistry, the alkoxysilaneshydrolase and condensate, yielding highly condensed silicon oxide around the supramicellar mesostructure. If a different inorganic composition is targeted, such as alumina or zirconia, the respective precursor should be added instead of alkoxysilanes.

It is also possible to obtain these mesoporous materials using a different path, which is based on coassembly with the inorganic precursor and the structure-directing agent. To achieve this, both the precursor and the surfactant should be added at the same time and the mesophase formation and hydrolysis condensation will take place simultaneously. In any case, the final step of the synthetic process consists of removal of the surfactant from the product to yield a network of cavities within the inorganic framework. The surfactant can be removed through calcination at high temperatures or solvent extraction under softer conditions ${ }^{29}$ (Figure 1). Ordered mesoporous silicates are very promising materials for tunable drug delivery as they can either enhance or slow down release kinetics simply by modifying structural properties (for example, pore size) or surface properties (for example, surface polarity). ${ }^{33}$ Liu $\mathrm{J}$ et al. ${ }^{34}$ demonstrate a novel and facile approach to the synthesis of mesoporous silicas with large and tunable mesopores $(10-16 \mathrm{~nm})$ from mixtures of sodium silicate and tetraethoxysilane (TEOS) in acetic acid/sodium acetate (HAcNaAc) buffer solutions ( $\mathrm{pH} 4.4$ ) using P123 as the template. Highly ordered mesoporous silicas with pore diameters as large as $15 \mathrm{~nm}$ and a mesocellular foam with cage size ca. $14-36 \mathrm{~nm}$ could be successfully synthesized by simply adjusting the molar ratio of sodium silicate to TEOS. ${ }^{34}$ Tang $\mathrm{H}$ et al. ${ }^{35}$ proposed a facile and efficient strategy for synthesizing $\mathrm{pH}$-responsive nanocarriers with chitosan-poly (methacrylic acid) (CS-PMAA) shells and MSN cores via an in situ polymerization approach ${ }^{35}$ Representative surfactants are the triblock copolymer P123, F127 and cationic template CTAB, corresponding to mesoporous materials of SBA-15, SBA-16 and MCM-41, respectively. Morphology control, pore size tailoring and surface chemistry are the three main research interests in the preparation of mesoporous materials. These are also the crucial characteristics determining the application potential. Because the preparation of mesoporous silica is based on a sol-gel process, the particle size of mesoporous silica can be controlled more precisely during the synthesis process compared with mesoporous silicon,the size fraction of which is typically obtained through milling and subsequent sieving or centrifugation. ${ }^{36}$ Typical characterization of SBA-15 includes a combination of analysis methods that characterize both the particle and the pore structure. These methods include gas sorption analysis, $\mathrm{x}$-ray diffraction or small angle $\mathrm{x}$-ray scattering (SAXS) and imaging via field emission scanning and high-resolution transmission electron microscopy (FE-SEM and HRTEM). ${ }^{37}$ A novel approach was used to fabricate MSNs of different pore sizes. The pore size of MSNs can be modulated conveniently from 3 to $10 \mathrm{~nm}$ by controlling the duration of etching withanNaBH4 solution. These MSNs could provide a promising platform for delivering water-insoluble drugs, controlling the release rate of drugs such as paclitaxe $1^{38}$ and telmisartan. ${ }^{39}$

\section{Surface functionalization}

The surface of mesoporous silica materials can be modified by any of three methods: co-condensation (one-pot synthesis) ${ }^{40}$ the imprint coating method ${ }^{41}$ and grafting (post-synthesis modification). ${ }^{42}$ Because anorganoalkoxysilane is introduced to a basic, aqueous CTAB-and-TEOS solution during condensation, the method is called the co-condensation functionalization method. ${ }^{43}$ Amit Dubey et al. ${ }^{44}$ developed the mild acidic nanocatalyst, ordered mesoporous silica, SBA -15 was functionalized with chloroacetic acid (SBA/CA) via post synthetic grafting technique for useful acidic conversions such as Knoveneganel condensation, Biginelli synthesis and Mannich reaction. ${ }^{44}$ Perez-Quintanilla D et al. ${ }^{45}$ reported that they used two mesoporous silicas (SBA-15 and MCM-41) to prepare solid phases modified with 2-mercaptopyridine through two different approaches (homogeneous and heterogeneous routes). They also described their application in a batch method for adsorption of $\mathrm{Hg}$ (II) from aqueous solutions. ${ }^{45}$ Mesoporous silica is shattered by sonication, resulting in an improved intramesoporous structure. With sonication-shattered mesoporous silica, a much higher protein loading density can be achieved, which may allow more sustained protein drug release. ${ }^{46}$

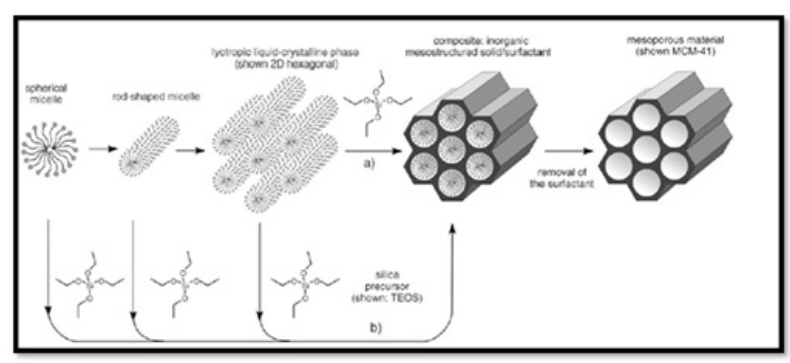

Figure I Formation of mesoporous material by liquid - crystal templating mechanism. ${ }^{32}$

\section{Methods of Drug Loading for MSNs}

The silica surface can be functionalized with organic groups to encourage greater drug loading by creating stronger bonding between the silica surface and the drug and to extend the release of the drug. The loading method can influence the properties of the drug, including the solid state structure and release rate. Fenofibrate, a drug that is poorly soluble in water, was loaded onto SBA-15 using the following methods:

a. Physical mixing,

b. Melting,

c. Solvent impregnation,

d. The liquid $\mathrm{CO}_{2}$ method and

e. The supercritical $\mathrm{CO}_{2}$ method.

The obtained results described in (Table 1). All the processing methods enhanced the release of the drug compared with the unprocessed drug. ${ }^{47}$ Coating drug-loaded core particles with a polymer film is one of the most widely investigated technologies in making multi-particulate dosage forms. A large variety of materials, such as Nonpareil ${ }^{\circledR}$ sugar beads and Celphere $\AA$ crystalline cellulose, have been used as core particles in this technology. However, the drug-loading ratio is relatively low since the loading area is limited to the outer surface of the core particles. Interest in overcoming these pharmaceutical difficulties through the use of porous materials as the drug-loading core and making use of their high surface area has been increasing. Drug-loaded particles appear to be particularly suited for controlled release and drug targeting. These systems are expected to enhance the bioavailability of poorly absorbed drugs, entailing a lowering of the therapeutic dose.

\section{Simple mixing}

An adsorbent was placed in a solution of the drug and stirred for a suitable time using a magnetic stirrer. The solution was then allowed 
to stand for 1 hour and the drug was separated and dried over 24 hours at $60^{\circ} \mathrm{C}$. This method is used for a variety of drugs including ibuprofen, dexamethasone, griseofulvin, ranitidine and furosemide.

Table I Drug loading methods and their results

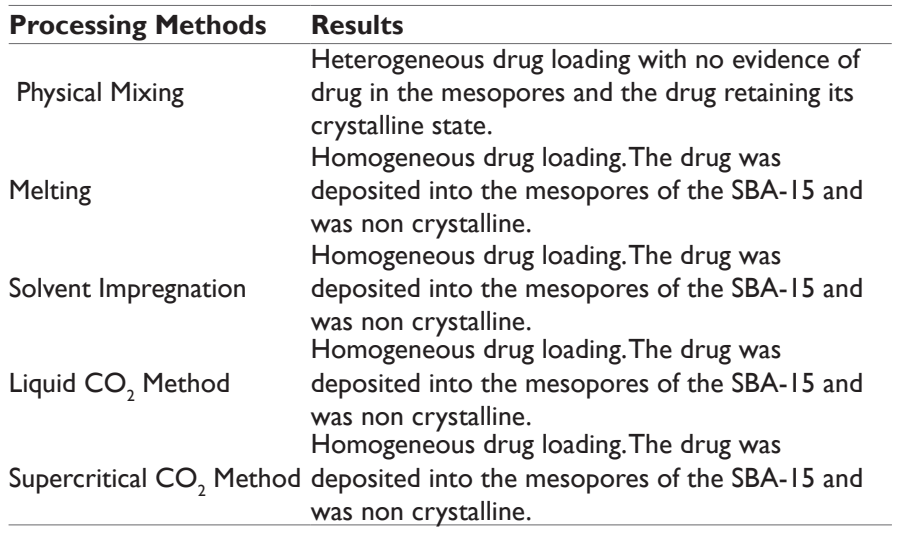

\section{Solvent evaporation}

The particle size of the adsorbent was restricted to $250-350 \mu \mathrm{m}$ by sieving to nullify the effects of variations. The drug was loaded in the solvent, after which the adsorbent was continuously added. The solution was kept at ambient conditions and the solvent was allowed to evaporate.

\section{Loading under high pressure}

The drug and the adsorbent were mixed in an appropriate ratio and the mixture kept in high-pressure adsorption equipment for a period of over 24 hours. The powder was washed with deionized water to get rid of unentrapped drug and dried in a vacuum oven at $65^{\circ} \mathrm{C}$ for 5 hours. This method is used for loading Brilliant Blue.

\section{Vacuum process}

The adsorbent was placed in the drug solution and the mixer evacuated for a suitable time, after which the vacuum was released. The adsorbent and drug solution were then allowed to stand for 1 hour. Then the solids were separated using a filter paper and dried for 24 hours at $60^{\circ} \mathrm{C}$. Various drugs including diltiazem hydrochloride, benzoic acid and sodium benzoate loaded on adsorbents using this method. In another method, the drug and adsorbent are mixed in a suitable volatile solvent for 6 hours and the solvent is evaporated under reduced pressure from the mixture obtained. The powder obtained was dried in vacuum for 3 hours. This method is used for loading hydrophobic drugs such as phytonadione.

\section{Stirring in drug solution or suspension}

In this method, the adsorbent is stirred into the drug solution or suspension, after which the mixture is dried in a simple tray drier. An excess of the drug solution or suspension is not required. The minimum required amount of drug solution is loaded. Therefore a vacuum process is not essential and a high yield can be achieved. This method can be used for a variety of drugs such as theophylline.

\section{Layer-by-layer (LbL) adsorption}

This technique usually involves the use of an aqueous solution at room temperature and thus it is suitable for encapsulating polypeptides and proteins drugs of poor stability. An LbL assembly of two oppositely charged polyelectrolytes at solid surfaces was developed as the alternating adsorption of these polyelectrolytes on a charged substrate due to their electrostatic attraction and the complex formation resulting in the defined macromolecular layer on the surfaces. ${ }^{7}$

\section{Pharmaceutical Applications of MSNs}

\section{Improving dissolution}

Mesoporous materials offer a potential means of increasing the dissolution of poorly soluble drugs ${ }^{48}$ via effects on the surface area or crystallinity. With their pore sizes being only a few times larger than the size of the drug molecule, the formation of a crystalline material is restricted by the confined space of the pores. Thus the drug retains its noncrystalline, amorphous form. The amorphous form is known to exhibit higher dissolution rates compared with the crystalline phase. $^{7}$ The use of mesoporous silicate MCM-41 to increase the dissolution rate of piroxicam a nonsteroidal anti-inflammatory class II (Biopharmaceutic Classification System) drug. ${ }^{49}$ Nasir Vadia et al. developed methotrexate-loaded mesoporous MCM-41 nanoparticles for improving the dissolution of methotrexate. ${ }^{50}$ Anna Kiwilsza et al. ${ }^{51}$ described SBA-15 and MCM-41 as promising carriers for achieving amorphous forms and enhanced dissolution rates for a nonionizable, poorly water-soluble drug, nimodipine.$^{52}$ Applicability of the ordered mesoporous silicate SBA-15 as an excipient to enhance dissolution, for a test series of 10 poorly soluble compounds with a high degree of physicochemical diversity (carbamazepine, cinnarizine, danazol, diazepam, fenofibrate, griseofulvin, indomethacin, ketoconazole, nifedipine and phenylbutazone). ${ }^{52}$ Zhang $\mathrm{Y}$ et al. ${ }^{53}$ developed MSNs loaded with a drug that is poorly soluble in water (telmisartan (TEL)) to improve the dissolution rate and enhance the drug-loading capacity for oral administration. ${ }^{53}$ SBA-15 enhanced the dissolution rate of furosemide. SBA-15, with its large surface area, can adsorb drug molecules by forming weak interactions that can be easily broken in biological fluids, allowing rapid drug release. ${ }^{54}$

\section{Chemical and pharmaceutical purification}

Adsorption in carbonaceous adsorbents is suitable for decolorization and purification of a wide range of organic and inorganic compounds including amines, hydrochloric and other mineral acids, amino acids, glycols and hydrocarbons. Catalysts can be poisoned or fouled by low-concentration organic compounds, sulfur, or mercury species. Carbonaceous materials, of both non impregnated and impregnated types, are typically used as "guard beds" to protect the catalysts (from fouling and/or deactivation) and the equipment (from corrosion) in streams such as natural gas, acetylene, ethane, propane and ethylene oxide.

\section{Purification and decolorization of food}

Adsorption in carbonaceous adsorbents is widely used for decolorization of natural and synthetic sweeteners, cane sugar syrup and vitamins and purification of glycerin.

\section{Improving bioavailability}

Low-density porous carriers such as porous silicon dioxide (Sylysia), polypropylene foam powder (Accurel), porous calcium silicate (Florite), magnesium aluminometasilicate (Neusilin) and porous ceramic, with open or closed pore structures, provide large surface areasand are used for improving the dissolution and bioavailability of poorly soluble drugs such as meloxicam, aspirin and indomethacin. ${ }^{7}$ Randy Mellaerts et al. ${ }^{55}$ reported that ordered mesoporous silica (OMS) is a promising carrier for achieving enhanced oral bioavailability of drugs (itraconazole) with poor aqueous solubility. ${ }^{55}$ Yanzhuo Zhang et al. ${ }^{56}$ investigated the effects of 
spherical MSNs as an oral drug delivery system (DDS) to improve the oral bioavailability of the model drug TEL and examined their cellular uptake and cytotoxicity. ${ }^{56}$

\section{Improving solubility}

Florite RE (FLR) is a porous calcium silicate that has many interparticle and intraparticle pores, particularly of sizes 12 and $0.15 \mu \mathrm{m}$, respectively, on its surface. FLR is easily dispersible in all aqueous fluids and has been used to adsorb oily and other drugs, as a compressive agent in pharmaceuticals and to improve solubility.

\section{Improving surface affinity}

Otsuka et al investigated the surface-modification of silica gel with silane coupling to improve the surface affinity to an oily medicine, phytonadione. However, a rapid release, especially an initial burst release, has been observed in some cases when inorganic porous particles have been used as the drug host.

\section{Sustained/controlled release}

Several porous minerals have been used, including synthetic zeolite, silica xerogel, porous hollow silica nanoparticles, porous hydroxyapatite, porous silica-calcium phosphate composites, porous calcium carbonate microparticles and other porous ceramics. These materials possess vast numbers of nanopores that allow the inclusion of drugs in them and are useful in making sustained/controlled release formulations. Accurel MP 1000 is characterized by an open porous network with a pore size predominantly in the microporous and mesoporous range. This microporous adsorbent has been evaluated for the development of floating DDS and stem development. A singleunit floating DDS consisting of low-density microparticles was developed using a porous carrier material (foam powder), drug and polymer to increase the gastric residence time. The floating behavior of the low-density DDS could be combined successfully with accurate control of drug release. Ohta et al investigated the use of silica gel as core particles to design a simple preparation for a controlled delivery system with a high drug content. Drug loading was carried out by immersing the silica gel in a preheated drug solution or suspension. The drug-loaded silica gel was coated with hydroxypropylmethylcellulose (HPMC) and an aqueous dispersion of ethylcellulose (Aquacoat ${ }^{\circledR}$ ) to control the drug release. The LbL self-assembly technique has been a powerful tool for depositing polyelectrolyte multilayer films on various particles through alternating deposition of oppositely charged polyelectrolytes, mainly through electrostatic attraction. There are potential applications of LbL adsorption on porous carriers in developing controlled release cosmetics for delivering proteins such as bovine serum albumin, glucose oxidase, urease and superoxide dismutase as the depositing species to prepare bioactive coreshell particles. Ito et al developed a self-emulsifying drug delivery system using three kinds of adsorbent (microporous calcium silicate (Florite ${ }^{\mathrm{TM}} \mathrm{RE}$ ), magnesium aluminometasilicate (Neusilin ${ }^{\mathrm{TM}} \mathrm{US}_{2}$ ) and silicon dioxide (Sylysia ${ }^{\mathrm{TM}}$ 320)) with low-molecular-weight heparin (LMWH). Their results suggest that adsorbent systems are useful as oral solid delivery systems of poorly absorbable drugs such as LMWH. ${ }^{7}$ MSN-based double DDS for glucose-responsive controlled release of insulin and cyclic AMP. Stimuli-responsive controlled-release systems have attracted much attention for their potential applications in drug and gene delivery. In particular, surfacefunctionalized, end-capped MSN materials have been demonstrated to be efficient stimuli-responsive controlled-release systems having the advantageous "zero premature release" property. ${ }^{57}$

\section{Other applications}

Porous ceramics are bodies comprising three-dimensional arrays of hollow polygons known as cells. If the cells are interconnected, the porous ceramic is termed an open-cell porous ceramic, while if they are isolated from each other, it is termed a closed-cell porous ceramic. Cells can also be partly open or partly closed. These properties have found many applications including as catalyst supports, filters for molten metals and hot gases, refractory linings for furnaces and porous implants in the area of biomaterials. Crystalline microporous materials generally have a narrow pore size distribution. Thus a microporous material selectively allows some molecules to enter its pores and rejects other molecules that are either too large or have a shape that does not match the shape of the pore. A number of applications involving microporous materials utilize such size and shape selectivity. ${ }^{7}$ Laura $\mathrm{J}$ Waters et al. ${ }^{58}$ reported that inclusion of fenofibrate in a series of mesoporous silicas using microwave irradiation enhanced the drug release. ${ }^{58} \mathrm{M}$ Moritz \& M Laniecki ${ }^{59}$ described the use of mesoporous SBA-15 material as a carrier for papaverine hydrochloride. Papaverine hydrochloride is known to be a nonselective smooth muscle relaxant. The activity of this drug is based on the inhibition of cyclic adenosine monophosphate (cAMP) and cyclic guanosine monophosphate (cGMP) phosphodiesterases. This alkaloid can be used in vasospasmic diseases such as spasms associated with subarachnoid hemorrhage and erectile dysfunction and spasms of the alimentary channel. The aim of this study was to formulate a drug (tablets, granules and coated powders) based on SBA-15, papaverine and excipients and to achieve extended-release profiles of the active substance..$^{59}$ Xinyue Huang et al. ${ }^{60}$ investigated the suitability of a number of different MSN structures for carrying a drug cargo. ${ }^{60}$ Shunai Che et al.$^{61}$ described the enhanced release of the poorly soluble drug itraconazole loaded in ordered mesoporous silica. ${ }^{61}$ MSNs as a stable, biocompatible and effective therapeutic and diagnostic ("theranostic") agent for US-based breast cancer imaging, diagnosis and treatment. ${ }^{62}$ Notch signaling, a key regulator of stem cells, is frequently overactivated in cancer. It is often linked to aggressive forms of cancer that evade standard treatment, highlighting Notch as an exciting therapeutic target. Notch is in principle "druggable" by $\gamma$-secretase inhibitors (GSIs), inhibitory peptides and antibodies, but clinical use of Notch inhibitors is restricted by severe side effects and there is a demand for alternative cancertargeted therapy. Here, we present a novel approach, using imagable MSNs as vehicles for targeted delivery of GSIs to block Notch signaling. ${ }^{63}$ Drug delivery, magnetic resonance and fluorescence imaging, magnetic manipulation and cell targeting are simultaneously possible using a multifunctional MSN. Superparamagnetic iron oxide nanocrystals were encapsulated inside mesostructured silica spheres that were labeled with fluorescent dye molecules and coated with hydrophilic groups to prevent aggregation. Water-insoluble anticancer drugs were delivered into human cancer cells, and surface conjugation with cancer-specific targeting agents increased the uptake into cancer cells relative to that in noncancerous fibroblasts. The highly versatile multifunctional nanoparticles can potentially be used for simultaneous imaging and therapeutic applications. ${ }^{64}$

Recently, a commercial albumin-bound paclitaxel (PTX) nanocarrier (Abraxane) was approved as the first new drug for pancreatic ductal adenocarcinoma in almost a decade. PTX improves the pharmaceutical efficacy of the first-line pancreatic cancer drug gemcitabine (GEM) through suppression of the tumor stroma and inhibiting the expression of the GEM-inactivating enzyme cytidine deaminase (CDA). So some researchers have developed an effective 
lipid-coated MSN platform for synergistic delivery of Gemcitabine and paclitaxel to human pancreatic cancer ${ }^{65}$ Guilan Quan et al. ${ }^{66}$ demonstrated that lactosaminated MSNs are promising inorganic carrier systems for asialoglycoprotein receptor-targeted anticancer drug delivery ${ }^{66}$ MSNs are highly interesting for gene delivery applications. ${ }^{67}$

\section{Conclusion}

The true value of the mesoporous silica platform, the flexibility, control of the physico-chemical parameters and straightforward surface tailoring supports all of the above-discussed strategies. Today, the mesoporous silica drug delivery field holds more questions than answers although recent data highlights the medical applicability of the platform. The major strength of the technology the flexibility (one technology any disease) makes a more systematic, collaborative effort to bring the technology to the doors of the clinic worthwhile. Taking into account all these features, it can be concluded that MSN is a novel smart very promising material for drug delivery.

\section{Acknowledgments}

None.

\section{Conflicts of interest}

None

\section{References}

1. Norhasyimi R, Ahmad ZA, Abdul Rahman M. A Review: Mesoporous Santa Barbara Amorphous-15, Types, Synthesis and Its Applications towards Biorefinery Production. Am J Appl Sci. 2010;7(12):1579-1586.

2. Van Speybroeck M, Mellaerts R, Mols R, et al. Enhanced absorption of the poorly soluble drug fenofibrate by tuning its release rate from ordered mesoporous silica. Eur J Pharm Sci. 2010;41(5):623-630.

3. Fumiaki Uejo, Waree Limwikrant, Kunikazu Moribe, et al. Dissolution improvement of fenofibrate by melting inclusion in mesoporous silica. Asian Journal of Pharmaceutical Sciences. 2013;8(6):329-335.

4. Zeid A. AL Othman. A Review: Fundamental Aspects of Silicate Mesoporous Materials. Materials. 2012;5(12):2874-2902.

5. Bharti C, Nagaich U, Pal AK, et al. Mesoporous silica nanoparticles in target drug delivery system: A review. Int $J$ Pharm Investig. 2015;5(3):124-133

6. Katsuhiko Ariga, Ajayan Vinu, Yusuke Yamauchi, et al. Nanoarchitectonics for Mesoporous Materials. Bull Chem Soc Jpn. 2012;85(1):1-32.

7. Ahuja G, Pathak K. Porous Carriers for Controlled/Modulated Drug Delivery. Indian J Pharm Sci. 2009;71(6):599-607.

8. Esmaeili Bidhendi M, Nabi Bidhendi GR, Mehrdadi N, et al. Modified Mesoporous Silica (SBA-15) with Trithiane as a new effective adsorbent for mercury ions removal from aqueous environment. $J$ Environ Health Sci Eng. 2014;12:100.

9. Asaad F Hassan, Sally A Helmy, Ahmed Donia. MCM-41 for Meloxicam Dissolution Improvement: in vitro Release and in vivo Bioavailability Studies. J Braz Chem Soc. 2015;26(7):1-12.

10. Sarita Panage, Vishal Pande, Suraj Patil, et al. Design and synthesis of mesoporous silica for inclusion of poorly water soluble drug sertaconazole nitrate as a drug delivery platform. Der Pharmacia Lettre. 2014;6(4):159-168.

11. Yu-Chih Lin, Liang-Yi Lin, Ming-Yi Gao, et al. Mesoporous silica nanoparticles synthesized from liquid crystal display manufacturing extracts as a potential candidate for a drug delivery carrier: evaluation of their safety and biocompatibility. Int $J$ Nanomedicine. 2013;8:3833-3842.

12. Mohseni M, Gilani K, Mortazavi SA. Preparation and Characterization of Rifampin Loaded Mesoporous Silica Nanoparticles as a Potential System for Pulmonary Drug Delivery. Iran J Pharm Res. 2015;14(1):27-34.

13. Lu J, Li Z, Zink JI, et al. In vivo tumor suppression efficacy of mesoporous silica nanoparticles-based drug-delivery system: enhanced efficacy by folate modification. Nanomedicine. 2012;8(2):212-220.

14. Zhao L, Qin H, Wu R, et al. Recent advances of mesoporous materials in sample preparation. J Chromatogr A. 2012;1228:193-204.

15. Rafael HA, Rufino Nava, Peza-Ledesma CL, et al. SBA-15 Mesoporous Silica as Catalytic Support for Hydrodesulfurization Catalysts-Review. Materials. 2013;6(9):4139-4167.

16. Wang Y, Zhao Q, Hu Y, et al. Ordered nanoporous silica as carriers for improved delivery of water insoluble drugs: a comparative study between three dimensional and two dimensional macroporous silica. Int J Nanomedicine. 2013;8:4015-4031.

17. Wang Z, Chen B, Quan G, et al. Increasing the oral bioavailability of poorly water-soluble carbamazepine using immediate-release pellets supported on SBA-15 mesoporous silica. Int J Nanomedicine. 2012;7:5807-5818.

18. Vallet Regi M, Balas F, Arcos D. Mesoporous materials for drug delivery. Angew Chem Int Ed Engl. 2007;46(40):7548-7558.

19. Kwon S, Singh RK, Perez RA, et al. Silica based mesoporous nanoparticles for controlled drug delivery. J Tissue Eng. 2013;4:1-18.

20. Nasir vadia, Sadhana Rajput. Mesoporous material, MCM41: a new drug carrier. Asian J Pharm Clin Res. 2011;4(2):44-53.

21. Slowing I, Trewyn BG, Lin VS. Effect of Surface Functionalization of MCM-41-Type Mesoporous Silica Nanoparticles on the Endocytosis by Human Cancer Cells. J Am Chem Soc. 2006;128(46):14792-14793.

22. Christian A, Veronika W, Christoph B, et al. Multifunctional Mesoporous Silica Nanoparticles as a Universal Platform for Drug Delivery. Chem Mater. 2014;26(1):435-451.

23. Tatsuya N, Fuminori S, Hisashi M, et al. Mesoporous silica nanoparticles for 19F magnetic resonance imaging, fluorescence imaging, and drug delivery. Chem Sci. 2015;6:1986-1990.

24. Slowing II, Brian GT, Supratim G, et al. Mesoporous Silica Nanoparticles for Drug Delivery and Biosensing Applications. Adv Funct Mater. 2007;17(8):1225-1236.

25. Cheng-Yu Lai. Mesoporous Silica Nanomaterials Applications in Catalysis. J Thermodyn Catal. 2013;5(1):1-3.

26. Kempen PJ, Greasley S, Parker KA, et al. Theranostic Mesoporous Silica Nanoparticles Biodegrade after Pro Survival Drug Delivery and Ultrasound/Magnetic Resonance Imaging of Stem Cells. Theranostics. 2015;5(6):631-642.

27. Lu J, Liong M, Li Z, et al. Biocompatibility, Biodistribution, and DrugDelivery Efficiency of Mesoporous Silica Nanoparticles for Cancer Therapy in Animals. Small. 2010;6(16):1794-1805.

28. Tang F, Li L, Chen D. Mesoporous Silica Nanoparticles: Synthesis, Biocompatibility and DrugDelivery. AdvMater. 2012;24(12):1504-1534.

29. Maria Vallet-Regi. Mesoporous Silica Nanoparticles: Their Projection in Nanomedicine. ISRN Materials Science. 2012;608548:1-20.

30. Galo J de AA Soler-Illia, Eduardo L Crepaldi, David Grosso, et al. Block copolymer-templated mesoporous oxides. Curr Opin Colloid Interface Sci. 2003;8:109-126.

31. Meynen V, Cool P, Vansant EF. Verified synthesis of mesoporous materials. Microporous Mesoporous Mater. 2009;125:170-223. 
32. Hoffmann F, Cornelius M, Morell J, et al. Silica-Based Mesoporous Organic-Inorganic Hybrid Materials. Angew Chem Int Ed. 2006;45(20):3216-3251.

33. Ukmar T, Maver U, Planinsek O et al. Understanding controlled drug release from mesoporous silicates: Theory and experiment. $J$ Control Release. 2011;155:409-417.

34. Jian Liu, Qihua Yang, XS Zhao, Lei Zhang Pore size control of mesoporous silicas from mixtures of sodium silicate and TEOS. Microporous Mesoporous Mater. 2007;106(1-3):62-67.

35. Hongyan T, Jia Guo, Yang Sun et al. Facile synthesis of $\mathrm{pH}$ sensitive polymer-coated mesoporous silica nanoparticles and their application in drug delivery. Int J Pharm. 2011;421(2): 388-396.

36. $\mathrm{Xu} \mathrm{W,} \mathrm{Riikonen} \mathrm{J,} \mathrm{Lehto} \mathrm{VP} \mathrm{Mesoporous} \mathrm{systems} \mathrm{for} \mathrm{poorly} \mathrm{soluble}$ drugs. Int J Pharm. 2012;453(1):181-197.

37. Siefker J, Karande P, Coppens MO Packaging biological cargoes in mesoporous materials: opportunities for drug delivery. Expert Opin Drug Deliv. 2014;11(11):1781-1793.

38. Jia L, Shen J, Li Z et al. Successfully tailoring the pore size of mesoporous silica nanoparticles: Exploitation of delivery systems for poorly water-soluble drugs. Int J Pharm. 2012;439(1-2):81-91.

39. Borbane SA, Pande VV, Vibhute SK et al. Design and Fabrication of Ordered Mesoporous Alumina Scaffold for Drug Delivery of poorly Water Soluble Drug. Austin Therapeutics. 2015;2(1):1015.

40. Liangming Wei, Nantao $\mathrm{Hu}$, Yafei Zhang Synthesis of PolymerMesoporous Silica Nanocomposites. Materials 3(7):4066-4079.

41. Slowing II, Vivero-Escoto JL, $\mathrm{Wu} \mathrm{CW}$, et al. Mesoporous silica nanoparticles as controlled release drug delivery and gene transfection carriers. Adv Drug Deliv Rev. 2008;60(11):1278-1288.

42. Rosenholm JM, Lindén M. Towards establishing structure activity relationships for mesoporous silica in drug delivery applications. $J$ Control Release. 2008;128(2):157-164.

43. Trewyn BG, Slowing II, Giri S, et al. Synthesis and Functionalization of a Mesoporous Silica Nanoparticle Based on the Sol-Gel Process and Applications in Controlled Release. Acc Chem Res. 2007;40(9):846-853.

44. Amit Dubey, Divya Sachdev, Neel Mani Srivasatava. Synthesis, characterization and catalytic application of ordered mesoporous silica nanocomposites functionalized with chloroacetic acid (SBA-15/CA). Adv Mat Lett. 2013;4(1):39-45.

45. D Perez-Quintanilla, I del Hierro, M Fajardo, et al. Mesoporous silica functionalized with 2-mercaptopyridine: Synthesis, characterization and employment for $\mathrm{Hg}(\mathrm{II})$ adsorption. Microporous Mesoporous Mater. 2006;89(1-3):58-68

46. Lei C, Chen B, Li X, et al. Non-destructively shattered mesoporous silica for protein drug Delivery. Microporous Mesoporous Mater. 2013; 175:157-160.

47. Ahern RJ, Hanrahan JP, Tobin JM, et al. Comparison of fenofibratemesoporous silica drug-loading processes for enhanced drug delivery. Eur J Pharm Sci. 2013;50(3-4):400-409.

48. Thomas MJ, Slipper I, Walunj A, et al. Inclusion of poorly soluble drugs in highly ordered mesoporous silica Nanoparticles. Int J Pharm. 2013;387(1-2):272-277.

49. Ambrogi V, Perioli L, Marmottini F, et al. Improvement of dissolution rate of piroxicam by inclusion into MCM-41 mesoporous silicate. Eur J Pharm Sci. 2007;32(3):216-222.

50. Vadia N, Rajput S. Study on formulation variables of methotrexate loaded mesoporous MCM-41 nanoparticles for dissolution enhancement. Eur J Pharm Sci. 2012;45(1-2):8-18.
51. Anna K, Bartlomiej M, Kacper D, et al. Mesoporous drug carrier systems for enhanced delivery rate of poorly water-soluble drug: nimodipine. $J$ Porous Mater. 2015;22(3):817-829.

52. Van Speybroeck M, Barillaro V, Thi TD, et al. Ordered Mesoporous Silica Material SBA-15: A Broad-Spectrum Formulation Platform for Poorly Soluble Drugs. J Pharm Sci. 2009;98(8):2648-2658.

53. Zhang Y, Zhi Z, Jiang T, et al. Spherical mesoporous silica nanoparticles for loading and release of the poorly water-soluble drug telmisartan. $J$ Controlled Release. 2010;145(3):257-263.

54. Ambrogi V, Perioli L, Pagano C, et al. Use of SBA-15 for furosemide oral delivery enhancement. Eur J Pharm Sci. 2012;46(1-2):43-48.

55. Mellaerts R, Mols R, Jammaer JA, et al. Increasing the oral bioavailability of the poorly water soluble drug itraconazole with ordered mesoporous silica. Eur J Pharm Biopharm. 2008;69(1):223-230.

56. Yanzhuo Z, Jiancheng W, Xiaoyu B, et al. Mesoporous Silica Nanoparticles for Increasing the Oral Bioavailability and Permeation of Poorly Water Soluble Drugs. Mol Pharm. 2012;9(3):505-513.

57. Zhao Y, Trewyn BG, Slowing II, et al. Mesoporous Silica NanoparticleBased Double Drug Delivery System for Glucose Responsive Controlled Release of Insulin and Cyclic AMP. J AM Chem Soc. 2009;131(24):8398-8400.

58. Waters LJ, Hussain T, Parkes G, et al. Inclusion of fenofibrate in a series of mesoporous silicas using microwave irradiation. Eur J Pharm Biopharm. 2013;85(3):936-941.

59. Michal Moritz, Marek Laniecki. Application of SBA-15 mesoporous material as the carrier for drug formulation systems. Papaverine hydrochloride adsorption and release study. Powder Technol. 2012;230:106-111.

60. Xinyue Huang, Neil P Young, Helen E Townley. Characterization and Comparison of Mesoporous Silica Particles for Optimized Drug Delivery. Nanomater Nanotechnol. 2014;4(2):1-14

61. Xiao Liu, Shunai Che. Enhanced release of the poorly soluble drug itraconazole loaded in ordered mesoporous silica. Sci China Chem. 2015;58(3):401-410.

62. Milgroom A, Intrator M, Madhavan K, et al. Mesoporous silica nanoparticles as a breast-cancer targeting ultrasound contrast agent. Colloids Surf B Biointerfaces. 2014;116:652-657.

63. Mamaeva V, Rosenholm JM, Bate-Eya LT, et al. Mesoporous Silica Nanoparticles as Drug Delivery Systems for Targeted Inhibition of Notch Signaling in Cancer. Mol Ther. 2011;19(8):1538-1546.

64. Liong $\mathrm{M}, \mathrm{Lu} \mathrm{J}$, Kovochich $\mathrm{M}$, et al. Multifunctional Inorganic Nanoparticles for Imaging, Targeting, and Drug Delivery. ACS Nano. $2008 ; 2(5): 889-896$.

65. Meng H, Wang M, Liu H, et al. Use of a Lipid-Coated Mesoporous Silica Nanoparticle Platform for Synergistic Gemcitabine and Paclitaxel Delivery to Human Pancreatic Cancer in Mice. ACS Nano. 2015;9(4):3540-3557.

66. Quan G, Pan X, Wang Z, et al. Lactosaminated mesoporous silica nanoparticles for asialoglycoprotein receptor targeted anticancer drug delivery. J Nanobiotechnology. 2015;13:7.

67. Mamaeva V, Sahlgren C, Lindén M. Mesoporous silica nanoparticles in medicine-Recent advances. Adv Drug Deliv Rev. 2013;65(5):689-702. 